\title{
Transitive group actions: (im)primitivity and semiregular subgroups
}

\author{
István Kovács • Aleksander Malnič • \\ Dragan Marušič • Štefko Miklavič
}

Received: 5 March 2013 / Accepted: 27 August 2014 / Published online: 11 September 2014

(C) Springer Science+Business Media New York 2014

\begin{abstract}
The following problem is considered: if $H$ is a semiregular abelian subgroup of a transitive permutation group $G$ acting on a finite set $X$, find conditions for (non)existence of $G$-invariant partitions of $X$. Conditions presented in this paper are derived by studying spectral properties of associated $G$-invariant digraphs. As an essential tool, irreducible complex characters of $H$ are used. Questions of this kind arise naturally when classifying combinatorial objects which enjoy a certain degree of symmetry. As an illustration, a new and short proof of an old result of Frucht et al. (Proc Camb Philos Soc 70:211-218, 1971) classifying edge-transitive generalized Petersen graphs, is given.
\end{abstract}

Keywords Permutation group - Semiregular group - Transitive group action . (Im)primitivity · Graph

\section{Introductory and historic remarks}

A combinatorial approach can often provide useful insight in the study of various algebraic structures, for example, group actions and in particular permutation groups. On the other hand, when investigating structural properties of graphs admitting (transitive) group actions one often relies on results which are purely algebraic. Literature covering both aspects of this fruitful interplay is vast, see for example $[3-6,9,10,13,20,22,26,30]$ and the literature therein.

I. Kovács · A. Malnič · D. Marušič · Š. Miklavič $(\varangle)$

University of Primorska, Andrej Marušič Institute, Muzejski trg 2, 6000 Koper, Slovenia

e-mail: stefko.miklavic@upr.si

A. Malnič

University of Ljubljana, Pedagoška fakulteta, Kardeljeva ploščad 16, 1000 Ljubljana, Slovenia 
A particular case of such an interplay of algebraic and combinatorial concepts is dealt with in this paper, and is motivated by the concept of $B$-groups. Recall that a (finite) group $H$ is called a $B$-group if a primitive permutation group containing $H$ as a regular subgroup is necessarily doubly transitive. (A transitive permutation group $G$ is primitive if the two trivial partitions are the only block systems of $G$, here called $G$-invariant partitions; $G$ is doubly transitive provided it is transitive on ordered pairs of distinct elements from the underlying set.) As a generalization let us call $H$ an $m$-Bgroup if a primitive permutation group containing $H$ as a semiregular subgroup with $m$ orbits is necessarily doubly transitive. (A nontrivial permutation, and more generally a nontrivial permutation group, is semiregular if all of its orbits have equal size.) Of course, a 1-B-group is just a B-group. By the classical results of Schur and Wielandt [32, Theorems 25.3 and 25.6], cyclic groups of composite order and dihedral groups are $B$-groups. Of more recent results, we mention the classification of all abelian $B$ groups by Li [19], and the classification of all metacyclic groups of order $p q$, where $p$ and $q$ are distinct primes, by Dobson [4].

As for 2-B-groups, cyclic groups of prime order $p$ are of this kind. Namely, in view of the classification of finite simple groups, a primitive group of degree $2 p, p>5$ a prime, is necessarily doubly transitive [2]. We mention that primitive permutations groups containing an element with exactly two cycles (not necessarily of the same length) have been classified by Müller [27].

As a natural generalization of the original question of Burnside, we therefore propose the following problem.

Problem 1.1 Given a positive integer $m$, determine the class of (abelian) m-B-groups.

We remark that asking a transitive permutation group to contain a semiregular abelian subgroup as in Problem 1.1 is not that restrictive at all. Indeed, although not every transitive permutation group contains a semiregular subgroup (see for example [3]), it is believed that the automorphism group of a vertex-transitive digraph does contain such a subgroup (see [23, Problem 2.4]) and, more generally, that the same holds for every 2 -closed transitive permutation group [1]. Substantial evidence supporting these claims has been gathered thus far, see $[3,5,6,11-13,18,25]$.

In this paper, we develop a general framework within which the study of $m$-Bgroups becomes easier to grasp. Typically, a natural environment for building such a framework is graph-theoretic: the structure of a transitive permutation group $G$ containing a semiregular abelian subgroup $H$ is studied via a collection of $G$-invariant digraphs (orbital graphs) associated with $G$. (Recall that a $G$-invariant digraph on $X$ is a digraph $\Gamma$ with vertex set $X$ and arc set a subset of $X \times X$, admitting $G$ as subgroup of automorphisms.) Within this setting we discuss conditions which narrow down the possible candidates for nontrivial $G$-invariant partitions, thus paving the way for a more in-depth analysis of $m$-B-groups for small values of $m$, and possible classification of such groups at least for $m=2$.

From a graph-theoretic point of view, this line of research is motivated by the following situation, often encountered in problems regarding symmetry of graphs. Given a bicirculant, that is, a graph admitting a cyclic subgroup with two orbits of equal size, there are two essentially different possibilities forcing such a graph to be vertex-transitive. Either there exists an automorphism swapping setwise the two orbits, 
called hereafter a swap, or there exists an automorphism mixing the vertices of the two orbits, called hereafter a mixer. For example, the class of generalized Petersen graphs $G P(n, s)$ [10] contains examples of each of the four possibilities that may occur with this respect: first, $G P(7,2)$ is not vertex-transitive and hence without swaps and mixers; second, the prism $G P(3,1)$ has swaps, but no mixers; third, the dodecahedron $G P(10,2)$ has mixers, but no swaps; and finally, the cube $G P(4,1)$ has both swaps and mixers. It seems therefore natural to seek for (non)existence conditions for these two kinds of automorphisms in bicirculants. Of course, the existence of a mixer in a bicirculant means that the two orbits of the semiregular cyclic subgroup do not form an invariant partition of the full automorphism group. On the other hand, the existence of a swap in a bicirculant is equivalent to there being a transitive subgroup of automorphisms with the two orbits of the cyclic group in question forming an invariant partition for this group. Clearly, knowing which cyclic groups are 2-B-groups is of vital importance in the study of arc-transitive bicirculants. On the other hand, studying existence of mixers for bicirculants is essentially the first step in determining which cyclic groups are 2-B-groups and which are not.

Coming back to conditions for (non)existence of certain $G$-invariant partitions, the approach we use in this paper is to consider the group $G$ acting on eigenspaces of $G$ invariant digraphs. In order to formulate our main result, some remarks are in order. First, recall that in a vertex-transitive $G$-invariant digraph each vertex has the same number of in- and out-neighbors, to which we shall refer as the valency $\operatorname{val}(\Gamma)$ of $\Gamma$. Further, the partition of $X$ into $H$-orbits is an equitable partition for $\Gamma$, that is, given two orbits $X_{1}$ and $X_{2}$, the number of arcs in $\Gamma$ starting from a fixed vertex in $X_{1}$ and ending in a vertex in $X_{2}$ does not depend on the choice of the vertex in $X_{1}$ (see $\left[14\right.$, p. 76] for details). Denote this number by $m_{X_{1}, X_{2}}$. The quotient $\Gamma / H$ is the (multi)digraph with vertex set consisting of all $H$-orbits, and with exactly $m_{X_{1}, X_{2}}$ arcs from $X_{1}$ to $X_{2}$. Finally, the characteristic polynomial of $\Gamma / H$ divides the characteristic polynomial of $\Gamma$, see [14, Lemma 2.2(c)]. A simplified version of our main result can now be stated as follows.

Theorem 1.2 Let $G$ be a transitive permutation group on a finite set $X$ containing an abelian semiregular subgroup $H$. Further, let $\Gamma$ be a $G$-invariant digraph with an eigenvalue $\lambda$ which is not the valency of $\Gamma$. If the multiplicity of $\lambda$ as the eigenvalue of the quotient graph $\Gamma / H$ is the same as the multiplicity of $\lambda$ as the eigenvalue of $\Gamma$, then there exists an intransitive normal subgroup of $G$ containing $H$.

Observe that, in the particular case when $H$ has prime number of orbits, Theorem 1.2 gives that the orbits of $H$ coincide with the orbits of such an intransitive normal subgroup, and hence form a $G$-invariant partition.

Theorem 1.2 is proved in Sect. 5 (as a consequence of more general statement, see Theorem 5.1), after introducing the required machinery in Sects. 2-4. As an application we give in Sect. 6, an alternative proof of an old result classifying edge-transitive generalized Petersen graphs [10] (see Theorem 6.1). Theorems of this nature are usually proved either by an elementary, although technically rather involved, combinatorial approach $[10,24,28]$, or else by using a normal subgroup reduction approach, often involving the classification of finite simple groups as an essential ingredient, see for 
example $[8,9,19,20,26,29]$. Our proof of Theorem 6.1 uses the second approach. However, being based on the techniques of this paper, it does not rely on the classification of finite simple groups.

\section{$2 G$-invariant partitions}

Let $G$ be a permutation group acting transitively on a finite set $X$. Suppose that $G$ contains a semiregular subgroup $H$ with orbits $X_{i}, i \in\{1, \ldots, m\}$. In the next proposition, we are going to show that each $G$-invariant partition of $X$ has a particularly nice structure. To state our result we need the following definition. Let $\Delta$ be a partition of $\{1, \ldots, m\}$, and let $\phi:\{1,2, \ldots, m\} \rightarrow \operatorname{Sub}(H)$, where $\operatorname{Sub}(H)$ denotes the set of subgroups of $H$. The mapping $\phi$ is $\Delta$-compatible if $\phi(i)=\phi(j)$ whenever $i$ and $j$ belong to the same class of $\Delta$.

Proposition 2.1 Let $G$ be a transitive permutation group on a finite set $X$, and let $H$ be a semiregular abelian subgroup of $G$ with orbits $X_{i}, i \in\{1, \ldots, m\}$. Let $\mathcal{B}$ be a $G$-invariant partition of $X$. Then there exist some $\underline{x} \in X_{1} \times \cdots \times X_{m}$, a partition $\Delta$ of $\{1, \ldots, m\}$, and $a \Delta$-compatible mapping $\phi:\{1, \ldots, m\} \rightarrow \operatorname{Sub}(H)$ such that

$$
\mathcal{B}=\left\{\bigcup_{i \in T} x_{i}^{h \phi(i)} \mid T \in \Delta, h \in H\right\}
$$

Proof Define a relation $\sim$ on $\{1, \ldots, m\}$ by letting $i \sim j$ if and only if there exists some $B \in \mathcal{B}$ such that $B \cap X_{i} \neq \emptyset$ and $B \cap X_{j} \neq \emptyset$. This is an equivalence relation. It is clearly reflexive and symmetric. To see that it is also transitive, let $i \sim j$ and $j \sim k$. Then there are $B_{1}, B_{2} \in \mathcal{B}$ such that $B_{1} \cap X_{i} \neq \varnothing, B_{1} \cap X_{j} \neq \emptyset$ and $B_{2} \cap X_{j} \neq \emptyset$, $B_{2} \cap X_{k} \neq \varnothing$. Let $x \in B_{1} \cap X_{j}$ and $x^{\prime} \in B_{2} \cap X_{j}$. Since $X_{j}$ is an orbit of $H$, there is $h \in H$ such that $x^{\prime}=x^{h}$. As $\mathcal{B}$ is a $G$-invariant partition of $X$ we have $B_{1}^{h}=B_{2}$. But $X_{i}$ is also an orbit for $H$; hence $B_{1} \cap X_{i} \neq \varnothing$ implies $B_{1}^{h} \cap X_{i}^{h} \neq \varnothing$, that is, $B_{2} \cap X_{i} \neq \emptyset$. Since $B_{2} \cap X_{k} \neq \emptyset$, we have $i \sim k$.

Let $\Delta$ denote the partition of $\{1, \ldots, m\}$ induced by the equivalence classes of $\sim$. Note that in the proof of transitivity of $\sim$ we actually prove a stronger property: if for some $B \in \mathcal{B}$ and some $T \in \Delta$ we have $B \cap X_{i} \neq \emptyset$ for some $i \in T$, then $B \cap X_{i} \neq \varnothing$ for every $i \in T$. This allows us to make the following definition. For each $T \in \Delta$ set

$$
\mathcal{B}_{T}=\left\{B \in \mathcal{B} \mid B \cap X_{i} \neq \emptyset \text { for all } i \in T\right\},
$$

fix a block $B_{T} \in \mathcal{B}_{T}$, and for each $i \in T$ choose an element $x_{i} \in B_{T} \cap X_{i}$. Denoting by $K_{T}$ the setwise stabilizer of $B_{T}$ in $H$, let $\phi:\{1, \ldots, m\} \rightarrow \operatorname{Sub}(H)$ be the mapping defined by

$$
\phi(i)=K_{T}, \quad T \in \Delta, i \in T .
$$


We now show that

$$
\mathcal{B}=\left\{\bigcup_{i \in T} x_{i}^{h \phi(i)} \mid T \in \Delta, h \in H\right\}
$$

First, observe that for each $i \in T$ we have

$$
K_{T}=\left\{h \in H \mid x_{i}^{h} \in B_{T}\right\} .
$$

Next, let $B^{\prime}$ be an arbitrary block in $\mathcal{B}_{T}$ and let $i \in T$. Since $X_{i}$ is an $H$-orbit there exists $h \in H$ such that $B^{\prime}=B_{T}^{h}$. Therefore, as $H$ is abelian and $B_{T}=\bigcup_{i \in T} x_{i}^{K_{T}}$ we have $B^{\prime}=\bigcup_{i \in T} x_{i}^{h K_{T}}$. This completes the proof.

For later reference, we shall denote the description of $G$-invariant partition $\mathcal{B}$ in Proposition 2.1 by $\Pi(\underline{x}, \Delta, \phi)$.

Corollary 2.2 With notation and assumptions of Proposition 2.1, let the partition $\mathcal{B}=\Pi(\underline{x}, \Delta, \phi)$ be $G$-invariant. Then the following holds.

(i) If $N$ is the kernel of the action of $G$ on $\mathcal{B}$, then for each $j \in\{1, \ldots, m\}$ we have $H \cap N \leq \phi(j)$.

(ii) For $T, T^{\prime} \in \Delta$ and $j \in T, j^{\prime} \in T^{\prime}$ we have that if $|\phi(j)|=\left|\phi\left(j^{\prime}\right)\right|$ then $|T|=\left|T^{\prime}\right|$.

Proof To prove (i), pick $j \in\{1,2, \ldots, m\}$ and $T \in \Delta$ such that $j \in T$. Observe that if $h \in H \cap N$, then $x_{j}^{h} \in B_{T}$. This implies $h \in \phi(j)$, that is, $H \cap N \leq \phi(j)$.

To prove (ii), note that since all blocks of $\mathcal{B}$ are of the same size, we have $|\phi(j)||T|=$ $\left|\phi\left(j^{\prime}\right)\right|\left|T^{\prime}\right|$ for all $T, T^{\prime} \in \Delta$ and $j \in T, j^{\prime} \in T^{\prime}$. The result now follows.

\section{Eigenspaces of $G$-invariant digraphs}

Let $G$ be a transitive permutation group on a finite set $X$. An orbit of $G$ acting on $X \times X$ is called an orbital of $G$. A $G$-invariant digraph over $X$ is a digraph with $X$ as the set of vertices such that $G$ acts as a group of automorphisms of $X$. The set of arcs of a $G$-invariant digraph is necessarily a union of orbitals. In this section, we describe the eigenspaces of $G$-invariant digraphs in the case when $G$ has an abelian semiregular subgroup. The results of this section will be used in Sect. 4 where we analyze $G$-invariant partitions of $X$.

We start by introducing some notation. Let $Z$ be a nonempty finite set with a fixed linear ordering of its elements. By $V_{Z}$ we denote the $|Z|$-dimensional $\mathbb{C}$-vector space of row vectors over $\mathbb{C}$, with components of vectors indexed by the elements of $Z$ (relative to the chosen ordering of $Z$ ). In particular, we occasionally consider a complex valued function $f: Z \rightarrow \mathbb{C}$ as a vector $\mathbf{v}_{f} \in V_{Z}$ whose $z$ th component, $z \in Z$, is equal to $f(z)$. Similarly, by Mat $_{Z}$ we denote the algebra of $|Z| \times|Z|$ matrices, with columns and rows indexed by the elements of $Z$ (relative to the chosen ordering of $Z$ ).

Recall that for a subset $S$ of a group $H$, the Cayley digraph Cay $(H, S)$ is the digraph with vertex set $H$, and with an arc pointing from $x \in H$ to $y \in H$ whenever $y x^{-1} \in S$. 
Fixing a linear ordering $h_{1}, h_{2}, \ldots, h_{n}$ of elements of $H$ we let $A(\operatorname{Cay}(H, S)) \in \mathrm{Mat}_{H}$ denote the adjacency matrix of Cay $(H, S)$ whose $\left(h_{i}, h_{j}\right)$ th entry is 1 if $h_{j} h_{i}^{-1} \in S$, and is 0 otherwise. If $H$ is abelian, let $H^{*}$ denote the dual group of complex irreducible characters $\chi: H \rightarrow \mathbb{C}$. By [14, Lemma 9.2] and Remark below, $\mathbf{v}_{\chi}$ is an eigenvector of $A(\mathrm{Cay}(H, S))$; its corresponding eigenvalue is

$$
\chi(S)=\sum_{g \in S} \chi\left(g^{-1}\right)=\sum_{g \in S} \overline{\chi(g)},
$$

with the standard convention that $\chi(S)=0$ whenever $S=\emptyset$. Moreover, the spectrum of $A(\operatorname{Cay}(H, S))$ is the multiset $\left\{\chi(S) \mid \chi \in H^{*}\right\}$, and $\left\{\mathbf{v}_{\chi} \mid \chi \in H^{*}\right\}$ forms an orthogonal basis of $V_{H}$ relative to the dot product. Note that this basis does not depend on $S$.

Remark Since all group actions in this paper are on the right, we need to consider matrices acting on row vectors. It is for this reason that the eigenvalues of $\operatorname{Cay}(H, S)$ are as defined above. However, in the context of left actions on column vectors, see for example [14, Lemma 9.2], the eigenvalues are given by $\sum_{g \in S} \chi(g)$.

The above results can be stated in a more general setting. Let $G$ be a transitive permutation group on a finite set $X$ and let $H \leq G$ be a semiregular subgroup. Choose a fixed linear ordering $h_{1}, h_{2}, \ldots, h_{n}$ of elements of $H$ and a linear ordering $X_{1}, \ldots, X_{m}$ of the set $\mathfrak{X}$ of $H$-orbits on $X$, along with arbitrarily chosen points of reference $b_{i} \in X_{i}$ for each $i$. This naturally induces a linear ordering of $X$, namely

$$
b_{1}^{h_{1}}, \ldots, b_{1}^{h_{n}}, b_{2}^{h_{1}}, \ldots, b_{2}^{h_{n}}, \ldots, b_{m}^{h_{1}}, \ldots, b_{m}^{h_{n}} .
$$

Now let $\Gamma$ be a $G$-invariant digraph on $X$. For $x, y \in X$, we shall write $x \rightarrow_{\Gamma} y$ if there is an arc pointing from $x$ to $y$. For $1 \leq i, j \leq m$ define

$$
S_{i j}=\left\{h \in H \mid b_{i} \rightarrow_{\Gamma} b_{j}^{h}\right\} .
$$

The array $\mathbf{S}=\left[S_{i j} \mid 1 \leq i, j \leq m\right]$ is called the symbol of $\Gamma$ relative to $H$, the ordering of $\mathfrak{X}$, and the chosen points of reference $\left(b_{1}, b_{2}, \ldots, b_{m}\right)$. The reader may check that the adjacency matrix of $\Gamma$ (relative to the chosen orderings) is given by the block matrix

$$
A_{\mathbf{S}}=\left(\begin{array}{ccc}
A\left(\operatorname{Cay}\left(H, S_{11}\right)\right) & \cdots & A\left(\operatorname{Cay}\left(H, S_{1 m}\right)\right) \\
\vdots & \ddots & \vdots \\
A\left(\operatorname{Cay}\left(H, S_{m 1}\right)\right) & \cdots & A\left(\operatorname{Cay}\left(H, S_{m m}\right)\right)
\end{array}\right)
$$

In order to display $A_{\mathbf{S}}$ in a more compact form, let $E_{i j}$ be the elementary $m \times m$ matrix having the $(i, j)$ th entry equal to 1 and all other entries equal to 0 , and let $A_{i j}=A\left(\operatorname{Cay}\left(H, S_{i j}\right)\right)$. Then

$$
A_{\mathbf{S}}=\sum_{i, j=1}^{m} E_{i j} \otimes A_{i j}
$$


where $\otimes$ denotes the tensor (Kronecker) product of matrices. By [7, p. 107], $(A \otimes$ $B)(C \otimes D)=A C \otimes B D$; in particular, $A^{-1} \otimes B^{-1}=(A \otimes B)^{-1}$. For later use, observe that for any $x=b_{i}^{h_{j}} \in X$, the $x$ th component of the tensor product $\mathbf{u} \otimes \mathbf{v}_{f} \in V_{X}$, where $\mathbf{u}=\left(u_{1}, \ldots, u_{m}\right) \in \mathbb{C}^{m}$ and $f: H \rightarrow \mathbb{C}$, is given by

$$
\left(\mathbf{u} \otimes \mathbf{v}_{f}\right)_{x}=u_{i} f\left(h_{j}\right)
$$

Further, if $U$ and $V$ are subspaces of $\mathbb{C}^{m}$ and $V_{H}$, respectively, then $U \otimes V$ is the subspace of $V_{X}$ spanned by all $\mathbf{u} \otimes \mathbf{v}, \mathbf{u} \in U$ and $\mathbf{v} \in V$. Finally, for a character $\chi \in H^{*}$ we define the $m \times m$ matrix $\chi(\mathbf{S}) \in$ Mat $\mathfrak{X}$ by

$$
\chi(\mathbf{S})=\left(\begin{array}{ccc}
\chi\left(S_{11}\right) & \cdots & \chi\left(S_{1 m}\right) \\
\vdots & \ddots & \vdots \\
\chi\left(S_{m 1}\right) & \cdots & \chi\left(S_{m m}\right)
\end{array}\right)
$$

Note that $\chi_{0}(\mathbf{S})$, where $\chi_{0}$ is the principal character of $H$, gives us the adjacency matrix of the quotient (multi)digraph $\Gamma / H$ relative to the chosen ordering of $\mathfrak{X}$.

Proposition 3.1 With notation and assumptions above, let $G$ be a transitive permutation group on a finite set $X$ and let $H \leq G$ be a semiregular abelian subgroup of order $n$ with $m$ orbits on $X$. Then the spectrum of a $G$-invariant digraph $\Gamma$ on $X$ is equal to the union (counting multiplicities) of spectra of all $\chi(\mathbf{S}), \chi \in H^{*}$, where $\mathbf{S}$ is the respective symbol of $\Gamma$.

Proof The spectrum of $\Gamma$ is equal to the spectrum of $A_{\mathbf{S}}$. Recall that all blocks $A_{i j}=$ $A\left(\operatorname{Cay}\left(H, S_{i j}\right)\right)$ have a common orthogonal eigenvector basis $\left\{\mathbf{v}_{\chi} \mid \chi \in H^{*}\right\}$, and that the eigenvalue of $A_{i j}$ corresponding to $\mathbf{v}_{\chi}$ is equal to $\chi\left(S_{i j}\right)$.

Let us fix an ordering $\chi_{1}, \ldots, \chi_{n}$ of $H^{*}$. With respect to the ordered eigenbasis $\mathbf{v}_{\chi_{1}}, \ldots, \mathbf{v}_{\chi_{n}}$, the matrix $A_{i j}$ takes the diagonal form $D_{i j}=\operatorname{diag}\left(\chi_{1}\left(S_{i j}\right), \ldots, \chi_{n}\left(S_{i j}\right)\right)$, that is, $A_{i j}=P^{-1} D_{i j} P$ where $P$ is the $n \times n$ matrix with $\mathbf{v}_{\chi_{1}}, \ldots, \mathbf{v}_{\chi_{n}}$ as rows. Consequently,

$$
A_{\mathbf{S}}=\left(I_{m} \otimes P\right)^{-1}\left(\sum_{i, j=1}^{m} E_{i j} \otimes D_{i j}\right)\left(I_{m} \otimes P\right),
$$

where the matrix $I_{m}$ is the identity matrix of size $m$. The reader may check that there is a permutation matrix which establishes a similarity relation between

$$
\sum_{i, j=1}^{m} E_{i j} \otimes D_{i j} \text { and } \sum_{k=1}^{n} E_{k k} \otimes \chi_{k}(\mathbf{S}) .
$$

As the latter of the two matrices is block-diagonal, it is now obvious that the spectrum of $A_{\mathbf{S}}$ is equal to the union of spectra of all matrices $\chi_{k}(\mathbf{S})$.

To calculate the eigenvectors of $A_{\mathbf{S}}$, let $\lambda$ be an eigenvalue and let $W_{\lambda} \leq V_{X}$ denote the respective eigenspace. First, define the set 


$$
K_{\mathbf{S}, \lambda}=\left\{\chi \in H^{*} \mid \operatorname{det}\left(\chi(\mathbf{S})-\lambda I_{m}\right)=0\right\}
$$

of all characters $\chi \in H^{*}$ for which $\lambda$ is an eigenvalue of $\chi(\mathbf{S})$. For $\chi \in K_{\mathbf{S}, \lambda}$, let $V_{\lambda, \chi}$ be the $\lambda$-eigenspace of the matrix $\chi(\mathbf{S})$. Now the eigenspace $W_{\lambda}$ can be written as $W_{\lambda}=\bigoplus_{\chi \in K_{\mathbf{S}, \lambda}} V_{\lambda, \chi} \otimes\left\langle\mathbf{v}_{\chi}\right\rangle$. Indeed, choosing an orthogonal basis $\mathfrak{U}_{\lambda, \chi}$ of $V_{\lambda, \chi}$ we have the following proposition.

Proposition 3.2 With notation and assumptions above, if $\lambda$ is an eigenvalue of $\Gamma, a$ basis of $W_{\lambda}$ is given by

$$
\left\{\mathbf{u} \otimes \mathbf{v}_{\chi} \mid \chi \in K_{\mathbf{S}, \lambda}, \mathbf{u} \in \mathfrak{U}_{\lambda, \chi}\right\} .
$$

Proof Fix an ordering $\chi_{1}, \ldots, \chi_{n}$ of $H^{*}$, and recall (from the proof of Proposition 3.1) that $A_{\mathbf{S}}$ and $\sum_{k=1}^{n} E_{k k} \otimes \chi_{k}(\mathbf{S})$ are similar. Thus, if $\lambda$ is an eigenvalue of $A_{\mathbf{S}}$, the dimension of $W_{\lambda}$ is equal to the dimension of the corresponding $\lambda$-eigenspace of $\sum_{k=1}^{n} E_{k k} \otimes \chi_{k}(\mathbf{S})$. Since this $\lambda$-subspace is equal to $\bigoplus_{\chi_{k} \in K_{\mathbf{S}, \lambda}}\left\langle\mathbf{e}_{k}\right\rangle \otimes V_{\lambda, \chi_{k}}$, where $\left\{\mathbf{e}_{1}, \ldots, \mathbf{e}_{n}\right\}$ is the standard basis of $\mathbb{C}^{n}$, we have

$$
\operatorname{dim} W_{\lambda}=\sum_{\chi_{k} \in K_{\mathbf{S}, \lambda}} \operatorname{dim} V_{\lambda, \chi_{k}}
$$

To complete the proof, we need to find an appropriate basis of $W_{\lambda}$. First note that for $\chi \in K_{\mathbf{S}, \lambda}$ and $\mathbf{u} \in V_{\lambda, \chi}$ the vector $\mathbf{u} \otimes \mathbf{v}_{\chi}$ is a $\lambda$-eigenvector of $A_{\mathbf{S}}$. Indeed,

$$
\begin{aligned}
\left(\mathbf{u} \otimes \mathbf{v}_{\chi}\right) A_{\mathbf{S}} & =\left(\mathbf{u} \otimes \mathbf{v}_{\chi}\right)\left(\sum_{i, j=1}^{m}\left(E_{i j} \otimes A_{i j}\right)\right) \\
& =\sum_{i, j=1}^{m}\left(\mathbf{u} E_{i j} \otimes \mathbf{v}_{\chi} A_{i j}\right)=\sum_{i, j=1}^{m}\left(\mathbf{u} E_{i j} \otimes \chi\left(S_{i j}\right) \mathbf{v}_{\chi}\right) \\
& =\left(\mathbf{u} \sum_{i, j=1}^{m} \chi\left(S_{i j}\right) E_{i j}\right) \otimes \mathbf{v}_{\chi}=\mathbf{u} \chi(\mathbf{S}) \otimes \mathbf{v}_{\chi} \\
& =\lambda\left(\mathbf{u} \otimes \mathbf{v}_{\chi}\right) .
\end{aligned}
$$

As the set $\left\{\mathbf{u} \otimes \mathbf{v}_{\chi} \mid \chi \in K_{\mathbf{S}, \lambda}, \mathbf{u} \in \mathfrak{U}_{\lambda, \chi}\right\}$ contains exactly $\sum_{\chi \in K_{\mathbf{S}, \lambda}} \operatorname{dim} V_{\lambda, \chi}$ elements, it remains to show that it is linearly independent. To this end we show that the vectors of this set are pairwise orthogonal. Consider two distinct eigenvectors $\mathbf{u} \otimes \mathbf{v}_{\chi}$ and $\mathbf{u}^{\prime} \otimes \mathbf{v}_{\chi^{\prime}}$ where $\mathbf{u} \in \mathfrak{U}_{\lambda, \chi}$ and $\mathbf{u}^{\prime} \in \mathfrak{U}_{\lambda, \chi^{\prime}}$. Their dot product is

$$
\left\langle\mathbf{u} \otimes \mathbf{v}_{\chi}, \mathbf{u}^{\prime} \otimes \mathbf{v}_{\chi^{\prime}}\right\rangle=\left\langle\mathbf{u}, \mathbf{u}^{\prime}\right\rangle\left\langle\mathbf{v}_{\chi}, \mathbf{v}_{\chi^{\prime}}\right\rangle .
$$

If $\chi \neq \chi^{\prime}$, then $\left\langle\mathbf{v}_{\chi}, \mathbf{v}_{\chi^{\prime}}\right\rangle=0$. If $\chi=\chi^{\prime}$ and $\mathbf{u} \neq \mathbf{u}^{\prime}$, then $\left\langle\mathbf{u}, \mathbf{u}^{\prime}\right\rangle=0$ since $\mathfrak{U}_{\lambda, \chi}$ is an orthogonal basis for $V_{\lambda, \chi}$. Therefore $\left\langle\mathbf{u} \otimes \mathbf{v}_{\chi}, \mathbf{u}^{\prime} \otimes \mathbf{v}_{\chi^{\prime}}\right\rangle=0$. The proof is complete. 
Example 3.3 Let $\Gamma=Q_{3}$ be the 3-cube, and let $c \in \operatorname{Aut}\left(Q_{3}\right)$ be the central reflection switching antipodal vertices. Let $G \leq \operatorname{Aut}\left(Q_{3}\right)$ be a vertex-transitive subgroup containing $c$. For the semiregular subgroup $H \leq G$, we take $H=\{\mathrm{id}, c\}$. Let $X_{1}, X_{2}$, $X_{3}$, and $X_{4}$ be the vertex orbits of $H$. Identifying $H=\mathbb{Z}_{2}$ and choosing the points of reference $b_{j} \in X_{j}(j=1,2,3,4)$ in such a way that no two of them are adjacent, the respective symbol is given by $S_{j j}=\emptyset$ and $S_{i j}=\{1\}$, for $i \neq j$. The elements of the dual group $H^{*}$ are $\chi_{0}(j)=1$ and $\chi_{1}(j)=(-1)^{j}$, for $j \in \mathbb{Z}_{2}$.

By direct computation, we get that the eigenvalues of $\chi_{0}(\mathbf{S})$ are $3,-1,-1,-1$, and the eigenvalues of $\chi_{1}(\mathbf{S})$ are $-3,1,1,1$. Therefore,

$$
K_{\mathbf{S}, 3}=K_{\mathbf{S},-1}=\left\{\chi_{0}\right\} \text { and } K_{\mathbf{S}, 1}=K_{\mathbf{S},-3}=\left\{\chi_{1}\right\}
$$

The respective subspaces $V_{\lambda, \chi}$ are

$$
\begin{aligned}
& V_{3, \chi_{0}}=V_{-3, \chi_{1}}=\langle(1,1,1,1)\rangle \\
& V_{1, \chi_{1}}=V_{-1, \chi_{0}}=\langle(1,0,0,-1),(0,1,0,-1),(0,0,1,-1)\rangle .
\end{aligned}
$$

By Propositions 3.1 and 3.2, the spectrum of $Q_{3}$ is $\{3,1,1,1,-1,-1,-1,-3\}$, and the eigenspaces $W_{\lambda}$ are

$$
\begin{aligned}
W_{3} & =\langle(1,1,1,1,1,1,1,1)\rangle \\
W_{1} & =\langle(1,-1,0,0,0,0,-1,1),(0,0,1,-1,0,0,-1,1),(0,0,0,0,1,-1,-1,1)\rangle \\
W_{-1} & =\langle(1,1,0,0,0,0,-1,-1),(0,0,1,1,0,0,-1,-1),(0,0,0,0,1,1,-1,-1)\rangle \\
W_{-3} & =\langle(1,-1,1,-1,1,-1,1,-1)\rangle .
\end{aligned}
$$

\section{$4 G$-invariant partitions from linear actions}

With the notation of Sect. 3, we extend the action of $G$ on $X$ to an action of $G$ on the vector space of row vectors $V_{X}$ in a natural way by setting

$$
\left(\mathbf{w}^{g}\right)_{x}=\mathbf{w}_{x^{g}}, \quad x \in X, g \in G
$$

Note that $\mathbf{w}^{g}=\mathbf{w} P_{g}$, where $P_{g} \in$ Mat $_{X}$ is the permutation matrix defined by

$$
\left(P_{g}\right)_{x, y}=\left\{\begin{array}{ll}
1 & \text { if } x^{g}=y \\
0 & \text { otherwise }
\end{array} \quad x, y \in X\right.
$$

Let $\lambda$ be an eigenvalue of a $G$-invariant digraph $\Gamma$. Since $P_{g}$ commutes with the adjacency matrix $A_{\mathbf{S}}$, the eigenspace $W_{\lambda}$ is clearly invariant for the action of $G$ on $V_{X}$. Although $G$ acts on $V_{X}$ faithfully, this might not be the case with $G$ acting on $W_{\lambda}$. Denote by $N_{\lambda}$ the kernel of the action of $G$ on $W_{\lambda}$. The orbits of $N_{\lambda}$ clearly form a $G$-invariant partition $\mathcal{B}_{\lambda}$ of $X$. By Proposition 2.1,

$$
\mathcal{B}_{\lambda}=\Pi\left(\underline{x}, \Delta_{\lambda}, \phi_{\lambda}\right)
$$


for an appropriate choice of $\underline{x}=\left(x_{1}, x_{2}, \ldots, x_{m}\right), \Delta_{\lambda}$, and $\phi_{\lambda}$. Our goal in this section is to describe $\mathcal{B}_{\lambda}$ in terms of $\Delta_{\lambda}$ and $\phi_{\lambda}$.

We first describe the function $\phi_{\lambda}$. To this end we introduce the following notation. For an ordered basis $\mathfrak{U}_{\lambda, \chi}=\left(\mathbf{u}_{1}, \ldots, \mathbf{u}_{r}\right)$ of $V_{\lambda, \chi}$, we let $\mathfrak{U}_{\lambda, \chi}^{(k)}$ denote the vector of length $r$ whose $i$ th component is equal to the $k$ th component of $\mathbf{u}_{i} \in \mathfrak{U}_{\lambda, \chi}$.

Lemma 4.1 With notation and assumptions above, the following hold.

(i) $N_{\lambda} \cap H=\bigcap_{\chi \in K_{\mathbf{S}, \lambda}} \operatorname{Ker} \chi$.

(ii) The kernel of the action of $G$ on $\mathcal{B}_{\lambda}$ is equal to $N_{\lambda}$.

(iii) Let $i \in\{1, \ldots, m\}$ and $\chi \in K_{\mathbf{S}, \lambda}$. If $\mathfrak{U}_{\lambda, \chi}^{(i)} \neq 0$, then $\phi_{\lambda}(i) \leq K e r \chi$. In particular, if $\mathfrak{U}_{\lambda, \chi}^{(i)} \neq 0$ for all $\chi \in K_{\mathbf{S}, \lambda}$, then $\phi_{\lambda}(i)=N_{\lambda} \cap H$.

Proof First observe that

$$
\left(\mathbf{u} \otimes \mathbf{v}_{\chi}\right)^{h}=\chi\left(h^{-1}\right)\left(\mathbf{u} \otimes \mathbf{v}_{\chi}\right)
$$

for all $\chi \in K_{\mathbf{S}, \lambda}, \mathbf{u} \in V_{\lambda, \chi}$, and $h \in H$. Indeed, take an arbitrary $x=b_{i}^{h^{\prime}}$, where $h^{\prime} \in H$. Then

$$
\begin{aligned}
\left(\left(\mathbf{u} \otimes \mathbf{v}_{\chi}\right)^{h}\right)_{x} & =\left(\mathbf{u} \otimes \mathbf{v}_{\chi}\right)_{x^{h^{-1}}}=\left(\mathbf{u} \otimes \mathbf{v}_{\chi}\right)_{b_{i}^{h^{\prime} h^{-1}}} \\
& =u_{i} \chi\left(h^{\prime} h^{-1}\right)=\chi\left(h^{-1}\right)\left(u_{i} \chi\left(h^{\prime}\right)\right) \\
& =\chi\left(h^{-1}\right)\left(\mathbf{u} \otimes \mathbf{v}_{\chi}\right)_{x} .
\end{aligned}
$$

By Proposition 3.2, we have that $h \in H$ belongs to $N_{\lambda}$ if and only if $\left(\mathbf{u} \otimes \mathbf{v}_{\chi}\right)^{h}=\mathbf{u} \otimes \mathbf{v}_{\chi}$ for each $\chi \in K_{\mathbf{S}, \lambda}$ and $\mathbf{u} \in V_{\lambda, \chi}$. Hence $h \in N_{\lambda}$ if and only if $\overline{\chi(h)}=\chi\left(h^{-1}\right)=1$ for all $\chi \in K_{\mathbf{S}, \lambda}$, or equivalently, $h \in \bigcap_{\chi \in K_{\mathbf{S}, \lambda}} \operatorname{Ker} \chi$. Part (i) follows.

To show (ii), let $g \in G$ acting on $X$ preserve the orbits in $\mathcal{B}_{\lambda}$. Recall that by the definition of $N_{\lambda}$ the coordinates of $\mathbf{w} \in W_{\lambda}$, indexed by the elements from the same orbit of $N_{\lambda}$, are the same. Thus, $\mathbf{w}_{x^{g}}=\mathbf{w}_{x}$ for all $\mathbf{w} \in W_{\lambda}$ and $x \in X$, and so $\left(\mathbf{w}^{g}\right)_{x}=\mathbf{w}_{x^{g}}=\mathbf{w}_{x}$. Consequently, $\mathbf{w}^{g}=\mathbf{w}$ and hence $g \in N_{\lambda}$. As the kernel of the action of $G$ on $\mathcal{B}_{\lambda}$ contains $N_{\lambda}$, the result follows.

In order to show (iii), note that since $\mathfrak{U}_{\lambda, \chi}^{(i)} \neq 0$, there exists $\mathbf{u} \in V_{\lambda, \chi}$ with $u_{i} \neq 0$. Let $B$ denote the block of $\mathcal{B}_{\lambda}$ containing $x_{i}$. Recall from the proof of Proposition 2.1 that $\phi_{\lambda}(i)=\left\{h \in H \mid x_{i}^{h} \in B\right\}$. Fix $h \in \phi_{\lambda}(i)$. Since $x_{i}$ and $x_{i}^{h}$ are in the same $N_{\lambda}$-orbit, the $x_{i}$ th and $x_{i}^{h}$ th coordinate of $\mathbf{u} \otimes \mathbf{v}_{\chi}$ are the same. As $x_{i}=b_{i}^{h^{\prime}}$ for some $h^{\prime} \in H$ we find that $u_{i} \chi\left(h^{\prime}\right)=u_{i} \chi\left(h^{\prime} h\right)$. Now $u_{i} \neq 0$ implies $\chi(h)=1$, and consequently $\phi_{\lambda}(i) \leq \operatorname{Ker} \chi$. Now if $\mathfrak{U}_{\lambda, \chi}^{(i)} \neq 0$ for every $\chi \in K_{\mathbf{S}, \lambda}$, we get $\phi_{\lambda}(i) \leq \bigcap_{\chi \in K_{\mathbf{S}, \lambda}} \operatorname{Ker} \chi=N_{\lambda} \cap H$, see (i). But since also $N_{\lambda} \cap H \leq \phi_{\lambda}(i)$, see Corollary 2.2(i), the claim is proved.

Following [31], for subsets $L \subseteq H$ and $L^{*} \subseteq H^{*}$ we define

$$
L^{\perp}=\left\{\chi \in H^{*} \mid L \subseteq \operatorname{Ker} \chi\right\} \text { and } L^{* \perp}=\bigcap_{\chi \in L^{*}} \operatorname{Ker} \chi .
$$


Observe that $L^{\perp}=\langle L\rangle^{\perp}$ and $L^{* \perp}=\left\langle L^{*}\right\rangle^{\perp}$. Moreover, $\perp$ is an anti-isomorphism between the subgroup lattices of $H$ and $H^{*}$. In this notation, Lemma 4.1(i) can be restated as

$$
N_{\lambda} \cap H=\left\langle K_{\mathbf{S}, \lambda}\right\rangle^{\perp}
$$

We now examine the partition $\Delta_{\lambda}$. Since $\Delta_{\lambda}$ is not readily at hand, we introduce certain auxiliary partitions of $\{1, \ldots, m\}$ which are easier to compute, and, as it will become clear shortly, they all have $\Delta_{\lambda}$ as a refinement. Hence their intersection gives at least some information about $\Delta_{\lambda}$, the more so when the former is "close" to the trivial partition $\Delta_{I}$ consisting of singletons, and giving no information if this intersection is the partition $\Delta_{U}$ consisting of the whole set.

For $\chi \in K_{\mathbf{S}, \lambda}$ choose an ordered basis $\mathfrak{U}=\mathfrak{U}_{\lambda, \chi}$ of $V_{\lambda, \chi}$. Let $\sim_{\lambda, \chi}$ be the equivalence relation on $\{1, \ldots, m\}$ defined by

$$
i \sim_{\lambda, \chi} j \Longleftrightarrow \mathfrak{U}^{(i)}=\chi(h) \mathfrak{U}^{(j)} \text { for some } h \in H .
$$

Note that this relation does not depend on the chosen basis $\mathfrak{U}$. We define the auxiliary partition $\Delta_{\lambda, \chi}$ as the equivalence classes of the relation $\sim_{\lambda, \chi}$.

Lemma 4.2 With notation and assumptions above, for $\chi \in K_{\mathbf{S}, \lambda}$ we have that $\Delta_{\lambda}$ is a refinement of $\Delta_{\lambda, \chi}$.

Proof Fix a character $\chi \in K_{\mathbf{S}, \lambda}$ and an ordered basis $\mathfrak{U}$ of $V_{\lambda, \chi}$. Let $i$ and $j$ be in the same partition class of $\Delta_{\lambda}$. Then there exists $h \in H$ such that $b_{i}$ and $b_{j}^{h}$ are in the same $N_{\lambda}$-orbit. Let $\mathbf{u}=\left(u_{1}, u_{2}, \ldots, u_{m}\right) \in \mathfrak{U}$. By Proposition 3.2, $\mathbf{u} \otimes \mathbf{v}_{\chi} \in W_{\lambda}$. Therefore, its $b_{i}$ th and $b_{j}^{h}$ th coordinates are the same, implying $u_{i}=\chi(h) u_{j}$. Since $\mathbf{u} \in \mathfrak{U}$ was arbitrary, it follows that $\mathfrak{U}^{(i)}=\chi(h) \mathfrak{U}^{(j)}$. Hence $i$ and $j$ are in the same partition class of $\Delta_{\lambda, \chi}$, as required.

Let $\mathcal{B}_{\lambda}=\Pi\left(\underline{x}, \Delta_{\lambda}, \phi_{\lambda}\right)$ be the $G$-invariant partition arising as orbits of the normal subgroup $N_{\lambda} \leq G$. Recall from the proof of Proposition 2.1 that one cannot determine $\underline{x}$ without knowing $\mathcal{B}_{\lambda}$. In contrast to this, $\Delta_{\lambda}$ and $\phi_{\lambda}$ can be computed-at least in certain cases - and this is sometimes even sufficient in order to completely reconstruct $\mathcal{B}_{\lambda}$. These remarks are illustrated in the next example.

Example 4.3 We continue with the analysis of $\Gamma=Q_{3}$ from Example 3.3. For each eigenvalue $\lambda$ of $Q_{3}$, we are going to find the invariant partition $\mathcal{B}_{\lambda}=\Pi\left(\underline{x}, \Delta_{\lambda}, \phi_{\lambda}\right)$ of the vertex set of $Q_{3}$.

By Lemma 4.1(iii), we have that $\phi_{\lambda}(i)=N_{\lambda} \cap H$ for each eigenvalue $\lambda$ and $i \in\{1,2,3,4\}$. Finding $N_{\lambda} \cap H$ is not a problem. Using Lemma 4.1(i), we obtain

$$
N_{3} \cap H=N_{-1} \cap H=H, \quad N_{-3} \cap H=N_{1} \cap H=\{0\} .
$$

In order to find $\Delta_{\lambda}$ we first compute the auxiliary partitions $\Delta_{\lambda, \chi}$. By definition, we easily get that

$$
\Delta_{1, \chi_{1}}=\Delta_{-1, \chi_{0}}=\Delta_{I}, \quad \Delta_{3, \chi_{0}}=\Delta_{-3, \chi_{1}}=\Delta_{U} .
$$


Therefore, by Lemma 4.2 we obtain that $\Delta_{1}=\Delta_{-1}=\Delta_{I}$. Consequently, $N_{1} \cap$ $H=\{0\}$ implies that $\mathcal{B}_{1}$ is the trivial block system consisting of singletons, and $N_{-1} \cap H=H$ implies that the blocks of $\mathcal{B}_{-1}$ are the orbits of $H$.

In the remaining two cases, direct analysis of the action of the group $G$ on $W_{\lambda}$ is needed in order to compute $N_{\lambda}$, and hence $\mathcal{B}_{\lambda}$. Consider $W_{3}=\langle(1,1,1,1,1,1,1,1)\rangle$. Then, obviously, $N_{3}=G$, and since $G$ is vertex-transitive the corresponding block system $\mathcal{B}_{3}$ is trivial with the whole set of vertices as the only block (and $\Delta_{3}$ is universal). Consider $W_{-3}=\langle(1,-1,1,-1,1,-1,1,-1)\rangle$. It follows that $N_{-3}$ is the largest subgroup in $G$ preserving the sets $\left\{b_{1}, b_{2}, b_{3}, b_{4}\right\}$ and $\left\{b_{1}^{1}, b_{2}^{1}, b_{3}^{1}, b_{4}^{1}\right\}$ (here $b_{i}^{1}$ denotes the image of the vertex $b_{i}$ under $1 \in H=\mathbb{Z}_{2}$ ); these two sets coincide with the bipartition sets of $Q_{3}$. Note that the group $N_{-3}$ has to be of index 2 in $G$, and therefore acts transitively on each of the two sets of bipartition. Hence, the corresponding block system $\mathcal{B}_{-3}$ coincides with the bipartition of $Q_{3}$ (and $\Delta_{-3}$ is the universal partition).

\section{Existence of imprimitivity block systems}

Recall from Proposition 3.1 that the spectrum of a $G$-invariant digraph $\Gamma$ is the union of spectra of all matrices $\chi(\mathbf{S}), \chi \in H^{*}$, where $\mathbf{S}$ is a symbol of $\Gamma$. Recall that the matrix $\chi_{0}(\mathbf{S})$ is nothing but the adjacency matrix of $\Gamma / H$. Also, the condition on the multiplicities of $\lambda$ as in Theorem 1.2 is equivalent to saying that $K_{\mathbf{S}, \lambda}=\left\{\chi_{0}\right\}$. This condition is further equivalent to saying that $H \leq N_{\lambda}$, where $N_{\lambda}$ is the kernel of the action of $G$ on the $\lambda$-eigenspace $W_{\lambda}$ (see Lemma 4.1(i))—which is in turn equal to the kernel of the action of $G$ on the orbits of $N_{\lambda}$ (see Lemma 4.1(ii)). Instead of Theorem 1.2 we now prove the following more general result.

Theorem 5.1 Let $G$ be a transitive permutation group on a finite set $X$ containing an abelian semiregular subgroup $H$. Further, let $\Gamma$ be a $G$-invariant digraph with symbol $\mathbf{S}$, and let $\lambda \neq \operatorname{val}(\Gamma)$ be an eigenvalue of $\Gamma$. Let $N_{\lambda}$ be the kernel of the action of $G$ on the $\lambda$-eigenspace $W_{\lambda}$. Then

(i) $N_{\lambda}$ contains $\left\langle K_{\mathbf{S}, \lambda}\right\rangle^{\perp}$.

(ii) If $\left\langle K_{\mathbf{S}, \lambda}\right\rangle<H^{*}$ is a proper subgroup, then the orbits of $N_{\lambda}$ form a nontrivial $G$-invariant partition of $X$.

Proof Part (i) follows directly from Lemma 4.1(i). For part (ii), let $\mathcal{B}_{\lambda}$ denote the $G$-invariant partition of $X$ consisting of the orbits of $N_{\lambda}$. By Proposition 2.1,

$$
\mathcal{B}_{\lambda}=\Pi\left(\underline{x}, \Delta_{\lambda}, \phi_{\lambda}\right)
$$

for an appropriate choice of $\underline{x}, \Delta_{\lambda}$, and $\phi_{\lambda}$. Since $\perp: \operatorname{Sub}(H) \rightarrow \operatorname{Sub}\left(H^{*}\right)$ is an involutory anti-isomorphism between the subgroup lattices of $H$ and $H^{*}$, we have that $\left\langle K_{\mathbf{S}, \lambda}\right\rangle^{\perp}$ is nontrivial. Hence $N_{\lambda}$ is nontrivial by (i), implying that $\mathcal{B}_{\lambda}$ does not consist of singletons.

We now show that $N_{\lambda}$ is intransitive. This is clear if $N_{\lambda} \cap H<H$. Suppose that $N_{\lambda} \cap H=H$ (which is equivalent to $K_{\mathbf{S}, \lambda}=\left\{\chi_{0}\right\}$, by Lemma 4.1(i)). By Corollary 2.2, we have that $\phi_{\lambda}(i)=H$ for all $i$. Since $\lambda \neq \operatorname{val}(\Gamma)$ it follows that 
$(1,1, \ldots, 1) \notin V_{\lambda, \chi_{0}}$. Thus, from the definition of $\Delta_{\lambda, \chi_{0}}$ it follows that $\Delta_{\lambda, \chi_{0}} \neq \Delta_{U}$. Hence $\Delta_{\lambda} \neq \Delta_{U}$, by Lemma 4.2 , and $N_{\lambda}$ is intransitive.

A transitive permutation group $G$ is called quasiprimitive if every nontrivial normal subgroup of $G$ is transitive. An immediate consequence of Theorem 5.1 is the following result dealing with quasiprimitive groups containing a semiregular abelian subgroup. A special case of Corollary 5.2, where $G$ is primitive and $H$ is regular, was proved by Knapp [17].

Corollary 5.2 Let $G$ be a quasiprimitive permutation group containing an abelian semiregular subgroup $H$, and let $\Gamma$ be a $G$-invariant digraph with symbol $\mathbf{S}$. If $\lambda \neq$ $\operatorname{val}(\Gamma)$ is an eigenvalue of $\Gamma$, then $\left\langle K_{\mathbf{S}, \lambda}\right\rangle=H^{*}$.

Generalizing the notion of a mixer of a bicirculant introduced in Sect. 1, we say that $g \in G$ is a mixer relative to $H$ (in short, a mixer when the subgroup $H$ is clear from the context), if the orbits of $H$ are not blocks of imprimitivity for $\langle g\rangle$. The following corollary is just a rephrasing of a special case covered in Theorem 5.1 into the mixers language.

Corollary 5.3 Let $G$ be a transitive permutation group on a finite set $X$ containing an abelian semiregular subgroup $H$ with a prime number of orbits, and let $\Gamma$ be a $G$-invariant digraph with symbol $\mathbf{S}$. If $G$ has a mixer relative to $H$, then for any eigenvalue $\lambda \neq \operatorname{val}(\Gamma)$ such that $\chi_{0} \in K_{\mathbf{S}, \lambda}$ we have $K_{\mathbf{S}, \lambda} \neq\left\{\chi_{0}\right\}$.

Proof Suppose, on the contrary, that $K_{\mathbf{S}, \lambda}=\left\{\chi_{0}\right\}$. Then $N_{\lambda}$ is intransitive, by Theorem 5.1(ii), and $N_{\lambda} \geq\left\langle\chi_{0}\right\rangle^{\perp}=H$, by Theorem 5.1(i). Therefore $\phi(i)=H$ for all $i$, and since $N_{\lambda}$ is intransitive, $\Delta_{\lambda} \neq \Delta_{U}$. As $H$ has prime number of orbits, it follows that $\Delta_{\lambda}=\Delta_{I}$, and $G$ has no mixers relative to $H$, a contradiction.

When the number of orbits of $H$ is relatively small, some additional information about mixers can be obtained using a more direct combinatorial approach which takes into account the intersections of orbits of $H$ with their images under a mixer. We here consider the case when $H$ has two orbits. Let $\Gamma$ be a $G$-invariant digraph (in this context also known as a bi-Cayley digraph) with symbol $\mathbf{S}$. For convenience we use abbreviations $S=S_{11}, T=S_{12}, Q=S_{21}$, and $R=S_{22}$. Note that $|S|=|R|$ and $|T|=|Q|$. Therefore, the eigenvalues of $\chi_{0}(\mathbf{S})$ are $\operatorname{val}(\Gamma)=|S|+|T|$ and $d(\Gamma)=|S|-|T|$. For $g \in G$, we define the subsets $L_{g}$ and $M_{g}$ of $H$ by

$$
\begin{aligned}
L_{g} & =\left\{h \in H \mid\left(b_{1}^{h}\right)^{g} \in X_{1}\right\}, \\
M_{g} & =\left\{h \in H \mid\left(b_{2}^{h}\right)^{g} \in X_{1}\right\} .
\end{aligned}
$$

Since $g$ is an automorphism we have $\left|L_{g}\right|+\left|M_{g}\right|=|H|$. Observe that $g$ is a swap if and only if $L_{g}=\emptyset$ and $M_{g}=H$, and that $g$ is a mixer if and only if $L_{g} \neq \varnothing$ and $L_{g} \neq H$.

In what follows, it will be convenient to view subsets of $H$ as elements of the group algebra $\mathbb{Q} H$. Following [32], a subset $A$ of $H$ is considered as $\underline{A}=\sum_{h \in A} h \in \mathbb{Q} H$. Characters of $H$ are naturally extended to an algebra homomorphism $\mathbb{Q} H \rightarrow \mathbb{C}$ by letting $\chi(\alpha)=\sum_{h \in H} a_{h} \chi(h)$ for $\alpha=\sum_{h \in H} a_{h} h$. Note that, given a subset $S \subseteq H$, we have $\chi(S)=\sum_{g \in S} \chi\left(g^{-1}\right)=\chi\left(\underline{S^{-1}}\right)$. 
Theorem 5.4 Let $G$ be a transitive permutation group containing an abelian semiregular subgroup $H$ having two orbits, and let $\Gamma$ be a $G$-invariant digraph. Then with notation and assumptions above, for all $g \in G$ and for all characters $\chi \in H^{*} \backslash K_{\mathbf{S}, d(\Gamma)}$ we have

$$
\chi\left(L_{g}\right)=\chi\left(M_{g}\right)=0 .
$$

Proof Let $g \in G$. For convenience we use a shorthand notation $L=L_{g}$ and $M=M_{g}$. Choose $h \in H$. The number of out-neighbors of $b_{1}^{h}$ that are contained in $b_{1}^{L} \cup b_{1}^{M}$ is equal to $|h S \cap L|+|h T \cap M|$. This number is equal to the coefficient of $h$ in $\underline{S^{-1}} \underline{L}+\underline{T^{-1}} \underline{M} \in \mathbb{Q} H$. Moreover, observe that it is also equal to the number of out-neighbors of $b_{1}^{h g}$ that are contained in $X_{1}$, which is either $|S|$ or $|Q|(=|T|)$, depending on whether $h \in L$ or $h \in H \backslash L$. Consequently,

$$
\underline{S^{-1}} \underline{L}+\underline{T^{-1}} \underline{M}=|S| \underline{L}+|T| \underline{H \backslash L}=d(\Gamma) \underline{L}+|T| \underline{H} .
$$

Similarly, by counting the number of out-neighbors of $b_{2}^{h}$ that are contained in $b_{1}^{L} \cup b_{1}^{M}$ (which equals the number of out-neighbors of $b_{2}^{h g}$ that are contained in $X_{1}$ ) we obtain the equation

$$
\underline{Q^{-1}} \underline{L}+\underline{R^{-1}} \underline{M}=|S| \underline{M}+|T| \underline{H \backslash M}=d(\Gamma) \underline{M}+|T| \underline{H} .
$$

Applying a non-principal character $\chi \in H^{*}$ to both of the above equalities we find that $\chi(\underline{L})$ and $\chi(\underline{M})$ are solutions of the following system of linear equations:

$$
\begin{aligned}
& \left(\chi\left(\underline{S^{-1}}\right)-d(\Gamma)\right) x+\quad \chi\left(\underline{\left.T^{-1}\right)} y=0\right. \\
& \chi\left(\underline{Q^{-1}}\right) x+\left(\chi\left(\underline{R^{-1}}\right)-d(\Gamma)\right) y=0 .
\end{aligned}
$$

If $\chi \notin K_{\mathbf{S}, d(\Gamma)}$ we have $\operatorname{det}(\chi(\mathbf{S})-d(\Gamma) I)=(\chi(S)-d(\Gamma))(\chi(R)-d(\Gamma))$ $-\chi(T) \chi(Q) \neq 0$. As the determinant of the above system is equal to $\operatorname{det}(\chi(\mathbf{S})-$ $d(\Gamma) I)$, it follows that $\chi(L)=\chi(M)=0$.

We remark that in the particular case when $H$ has two orbits, Corollary 5.3 follows directly from Theorem 5.4. Namely, let $g \in G$ be a mixer relative to $H$. Then $L_{g}$ and $M_{g}$ are nonempty proper subsets of $H$. It is well-known that if all non-principal characters vanish on a subset $A \subseteq H$, then either $A=\emptyset$ or $A=H$; see for instance [31, Corollary 1.3.5]. Therefore, there exists a non-principal character $\chi \in H^{*}$ such that either $\chi\left(L_{g}\right) \neq 0$ or $\chi\left(M_{g}\right) \neq 0$. By Theorem 5.4, we have $\chi \in K_{\mathbf{S}, d(\Gamma)}$.

\section{An application: generalized Petersen graphs}

To further illustrate the possible use of techniques developed here, we give an alternative short proof of a well-known classical result about edge-transitivity (and thus arc-transitivity) of generalized Petersen graphs due to Frucht et al. [10, Theorem 2]. Recall that the generalized Petersen graph $\mathrm{GP}(n, s)$ is the bicirculant relative to the 
cyclic group $H=\mathbb{Z}_{n}$ and admitting a symbol $\mathbf{S}$ with $S_{11}=\{1,-1\}, S_{12}=S_{21}=\{0\}$, and $S_{22}=\{s,-s\}$ where $s \in \mathbb{Z}_{n} \backslash\{0, n / 2\}$.

Theorem 6.1 A generalized Petersen graph $\Gamma=G P(n, s)$ is edge-transitive (and thus arc-transitive) if and only if $(n, s)$ is one of the following pairs: $(4,1),(5,2),(8,3),(10,2),(10,3),(12,5)$, or $(24,5)$.

As remarked in Sect. 1, theorems of this nature are usually proved either by an elementary, although technically rather involved, combinatorial approach (as, for instance, counting the number of eight cycles in the original proof of the above theorem), or else by using a two-step (normal subgroup) reduction approach. The first step identifies a small restricted family from which all other graphs in the class can be reconstructed. The actual constructions are then provided in the second step. In our proof we will make use of graph covers, and therefore we briefly review the necessary definitions and facts.

A covering projection of a graph $\widetilde{X}$ is a surjective mapping $p: \widetilde{X} \rightarrow X$ such that for each $\tilde{u} \in V(\tilde{X})$ the set of arcs emanating from $\tilde{u}$ is mapped bijectively onto the set of arcs emanating from $u=p(\tilde{u})$. The graph $\widetilde{X}$ is called a covering graph of the base graph $X$. The set $\mathrm{fib}_{u}=p^{-1}(u)$ is a fiber of a vertex $u \in V(X)$. The subgroup $K$ of all those automorphisms of $\tilde{X}$ which fix each of the fibers setwise is called the group of covering transformations. The graph $\tilde{X}$ is also called a $K$-cover of $X$. It is a simple observation that the group of covering transformations of a connected covering graph acts semiregularly on each of the fibers. In particular, if the group of covering transformations is regular on the fibers of $\tilde{X}$, we say that $\tilde{X}$ is a regular $K$-cover. We say that $\alpha \in$ Aut $X$ lifts to an automorphism of $\tilde{X}$ if there exists an automorphism $\tilde{\alpha} \in$ Aut $\tilde{X}$, called a lift of $\alpha$, such that $\tilde{\alpha} p=p \alpha$. If the covering graph $\tilde{X}$ is connected then $K$ is the lift of the trivial subgroup of Aut $X$. Note that a subgroup $G \leq \operatorname{Aut} \widetilde{X}$ projects if and only if the partition of $V(\tilde{X})$ into the orbits of $K$ is $G$-invariant.

A combinatorial description of a $K$-cover was introduced through a voltage graph by Gross and Tucker [16] as follows. Let $X$ be a graph and $K$ be a finite group. A voltage assignment of $X$ is a mapping $\zeta: A(X) \rightarrow K$ with the property that $\zeta(u, v)=\zeta(v, u)^{-1}$ for any arc $(u, v) \in A(X)$ (here, and in the rest of the paper, $\zeta(u, v)$ is written instead of $\zeta((u, v))$ for the sake of brevity). The voltage assignment $\zeta$ extends to walks in $X$ in a natural way. In particular, for any walk $D=u_{1} u_{2} \cdots u_{t}$ of $X$ we let $\zeta(D)$ denote the product voltage $\zeta\left(u_{1}, u_{2}\right) \zeta\left(u_{2}, u_{3}\right) \cdots \zeta\left(u_{t-1}, u_{t}\right)$ of $D$, that is, the $\zeta$-voltage of $D$. The values of $\zeta$ are called voltages, and $K$ is the voltage group. The voltage graph $X \times_{\zeta} K$ derived from a voltage assignment $\zeta: A(X) \rightarrow K$ has vertex set $V(X) \times K$, and edges of the form $\{(u, g),(v, \zeta(x) g)\}$, where $x=(u, v) \in A(X)$. Clearly, $X \times_{\zeta} K$ is a covering of $X$ with the first coordinate projection. By letting $K$ act on $V\left(X \times_{\zeta} K\right)$ as $(u, g)^{g^{\prime}}=\left(u, g g^{\prime}\right),(u, g) \in V\left(X \times_{\zeta} K\right), g^{\prime} \in K$, one obtains a semiregular group of automorphisms of $X \times_{\zeta} K$, showing that $X \times_{\zeta} K$ can in fact be viewed as a $K$-cover of $X$.

Given a spanning tree $T$ of $X$, the voltage assignment $\zeta: A(X) \rightarrow K$ is said to be $T$-reduced if the voltages on the tree arcs equal the identity element in $K$. In [15] it is shown that every regular covering graph $\tilde{X}$ of a graph $X$ can be derived from a $T$-reduced voltage assignment $\zeta$ with respect to an arbitrary fixed spanning tree $T$ of $X$. 
The problem of whether an automorphism $\alpha$ of $X$ lifts or not is expressed in terms of voltages as follows. Given $\alpha \in$ Aut $X$ and the set of fundamental closed walks $\mathcal{C}$ based at a fixed vertex $v \in V(X)$, we define $\bar{\alpha}=\left\{\left(\zeta(C), \zeta\left(C^{\alpha}\right)\right) \mid C \in \mathcal{C}\right\} \subseteq K \times K$. Note that if $K$ is abelian, $\bar{\alpha}$ does not depend on the choice of the base vertex, and the fundamental closed walks at $v$ can be substituted by the fundamental cycles generated by the cotree arcs of $X$. Also, from the definition, it is clear that for a $T$-reduced voltage assignment $\zeta$ the derived graph $X \times_{\zeta} K$ is connected if and only if the voltages of the cotree arcs generate the voltage group $K$. It was proved in [21, Theorem 4.2] that, given a connected regular cover $X \times_{\zeta} K$ of a graph $X$ derived from a voltage assignment $\zeta$ with the voltage group $K$, then an automorphism $\alpha$ of $X$ lifts if and only if $\bar{\alpha}$ is a function which extends to an automorphism of $K$.

Proof of Theorem 6.1 Our proof uses the above two-step approach. In the first part, we show that every edge-transitive generalized Petersen graph $\Gamma$ is a regular cyclic cover either of the cube or of the Petersen graph (such that $\operatorname{Aut}(\Gamma)$ projects). In the second part, we determine all such covers.

Part 1. First note that the eigenvalues of $\chi_{0}(\mathbf{S})$ are $\operatorname{val}(\Gamma)=3$ and $d(\Gamma)=1$. The respective eigenspace $V_{1, \chi_{0}}$ is spanned by $(1,-1)$. By the definition of the relation $\sim_{1, \chi_{0}}$ the partition $\Delta_{1, \chi_{0}}$ is equal to $\Delta_{I}$, and by Lemma 4.2 , the partition $\Delta_{1}$ is also equal to $\Delta_{I}$. Let $K=N_{1} \cap H$. By Lemma 4.1(iii), $\phi(1)=\phi(2)=K$. Thus, the $\operatorname{Aut}(\Gamma)$-invariant partition $\mathcal{B}_{1}$ of the vertex set of $\Gamma$, induced by the eigenvalue 1 , is just the partition formed by the orbits of $K$. Since the two orbits of $H$ are joined by one perfect matching, we get that any two orbits of $K$ are joined by at most one perfect matching. This implies that $N_{1}=K$ and $\Gamma$ is a normal cover of $\Gamma / K$.

In order to determine $K$, and consequently $\Gamma / K$, recall that $K=\left\langle K_{\mathbf{S}, 1}\right\rangle^{\perp}=$ $\cap \operatorname{Ker} \chi$, where $\chi$ ranges over all characters in $K_{\mathbf{S}, 1}$. Therefore, we first find $K_{\mathbf{S}, 1}$. The set $K_{\mathbf{S}, 1}$ is determined using the assumption that $\Gamma$ is edge-transitive, and hence that it has a mixer relative to $H$. By Corollary 5.3, the set $K_{\mathbf{S}, 1}$ contains a non-principal character, say $\chi$, implying that

$$
(\chi(1)+\chi(-1)-1)(\chi(s)+\chi(-s)-1)=1 .
$$

All solutions of (1) give all possible characters in $K_{\mathbf{S}, 1}$. (Observe that $\chi \in K_{\mathbf{S}, 1}$ if and only if $\chi^{-1} \in K_{\mathbf{S}, 1}$.) For a natural number $m$ let $\xi_{m}=e^{\frac{2 \pi \mathbf{i}}{m}}$. As $\chi(1)=\xi_{n}^{j}$ for some $j \in \mathbb{Z}_{n} \backslash\{0\}$, Eq. (1) rewrites as $\left(\xi_{n}^{j}+\xi_{n}^{-j}-1\right)\left(\xi_{n}^{j s}+\xi_{n}^{-j s}-1\right)=1$. By setting $j^{\prime}=j / \operatorname{gcd}(j, n), n^{\prime}=n / \operatorname{gcd}(j, n)$, and $s^{\prime} \equiv s\left(\bmod n^{\prime}\right)$, this last equation becomes

$$
\left(\xi_{n^{\prime}}^{j^{\prime}}+\xi_{n^{\prime}}^{-j^{\prime}}-1\right)\left(\xi_{n^{\prime}}^{j^{\prime} s^{\prime}}+\xi_{n^{\prime}}^{-j^{\prime} s^{\prime}}-1\right)=1
$$

Since $j^{\prime}$ and $n^{\prime}$ are coprime, $\xi_{n^{\prime}}^{j^{\prime}}$ and $\xi_{n^{\prime}}$ are both primitive $n^{\prime}$ th roots of unity. Hence their minimal polynomial is the same, implying that

$$
\left(\xi_{n^{\prime}}+\xi_{n^{\prime}}^{-1}-1\right)\left(\xi_{n^{\prime}}^{s^{\prime}}+\xi_{n^{\prime}}^{-s^{\prime}}-1\right)=1
$$


If both factors in (2) are positive then $n^{\prime}=1$, forcing $j=n$. However, this is not possible as $\chi$ is non-principal. Therefore, $\xi_{n^{\prime}}+\xi_{n^{\prime}}^{-1}-1=2 \cos \left(2 \pi / n^{\prime}\right)-1<0$, and so $n^{\prime} \leq 5$. A direct analysis yields that either $n^{\prime}=4$ and $s^{\prime}= \pm 1$, or else $n^{\prime}=5$ and $s^{\prime}= \pm 2$. It follows that $j^{\prime}= \pm 1$ in the first case while $j^{\prime} \in\{ \pm 1, \pm 2\}$ in the second case. Hence for some natural number $m$ either $n=4 m, j= \pm m$, and $s \equiv \pm 1$ $(\bmod 4)$, or else $n=5 m, j \in\{ \pm m, \pm 2 m\}$, and $s \equiv \pm 2(\bmod 5)$. We now analyze each of these cases separately.

Let $n=4 m, j= \pm m$, and $s \equiv \pm 1(\bmod 4)$, where $m$ is not divisible by 5 . Since $\chi(1)=\xi_{n}^{j} \in\left\{\xi_{4}, \xi_{4}^{-1}\right\}$ we have that $K_{\mathbf{S}, 1}=\left\{\chi_{0}, \chi, \chi^{-1}\right\}$, and so $K=\left\langle K_{\mathbf{S}, 1}\right\rangle^{\perp}$ is the unique index 4 subgroup in $\mathbb{Z}_{n}$. Since $s \equiv \pm 1(\bmod 4)$, the graph $\Gamma / K$ is the cube.

Let $n=5 m, j \in\{ \pm m, \pm 2 m\}$, and $s \equiv \pm 2(\bmod 5)$ where $m$ is not divisible by 4 . Since $\chi(1)=\xi_{n}^{j} \in\left\{\xi_{5}, \xi_{5}^{-1}, \xi_{5}^{2}, \xi_{5}^{-2}\right\}$ we have that $K_{\mathbf{S}, 1}=\left\{\chi_{0}, \chi, \chi^{-1}, \chi^{2}, \chi^{-2}\right\}$, and so $K=\left\langle K_{\mathbf{S}, 1}\right\rangle^{\perp}$ is the unique index 5 subgroup in $\mathbb{Z}_{n}$. Since $s \equiv \pm 2(\bmod 5)$, the graph $\Gamma / K$ is the Petersen graph.

Let $n=20 m$ where $s \equiv \pm 1(\bmod 4)$ and $s \equiv \pm 2(\bmod 5)$. By the above analysis, the group $\left\langle K_{\mathbf{S}, 1}\right\rangle=\left\langle\chi, \chi^{\prime}\right\rangle$ where $\chi(1)=\xi_{4}$ and $\chi^{\prime}(1)=\xi_{5}$. In this case, $K=$ $\left\langle K_{\mathbf{S}, 1}\right\rangle^{\perp}$ is the unique index 20-subgroup in $\mathbb{Z}_{n}$. Since $s \equiv \pm 1(\bmod 4)$ and $s \equiv \pm 2$ $(\bmod 5)$ we have, by the Chinese Remainder Theorem, that $s \equiv \pm 3(\bmod 20)$ or $s \equiv \pm 7(\bmod 20)$. Hence $\Gamma / K$ is either $\operatorname{GP}(20,3)$ or $\operatorname{GP}(20,7)$. In particular, $\Gamma / K$ and hence also $\Gamma$ is a regular cyclic cover of both the cube and the Petersen graph. (Actually, as it will become clear in Part 2, this case cannot occur.)

Note that in all of the above cases $K$ is isomorphic to $\mathbb{Z}_{m}$.

Part 2. Recall from Part 1 that $\Gamma \rightarrow \Gamma / K$ is a regular cyclic covering projection such that $\operatorname{Aut}(\Gamma)$ projects, and hence some minimal arc-transitive subgroup of $\Gamma / K$ lifts. We determine all such covers where the base graph is either the cube or the Petersen graph. Since GP(20,3) or GP(20,7) are not among them, the case where the base graph is one of these two need not be considered.

Let $Y$ be the cube. Denote its vertices by $\{0,1,2,3,4,5,6,7\}$ in such a way that 01230 and 45674 are the outer and the inner cycles, respectively, and 04, 15, 26, and 37 are the spokes. Choose a spanning tree consisting of edges 01, 12, 23, 04, 15, 26, and 37. The base homology cycles are $C_{1}=30123, C_{2}=45104, C_{3}=56215$, $C_{4}=67326$, and $C_{5}=7401237$. Voltages on the spanning tree are 0 . Without loss of generality, we can assume that the voltages on the remaining arcs are $\zeta(3,0)=1$, and $\zeta(4,5)=\zeta(5,6)=\zeta(6,7)=a$, and $\zeta(7,4)=a+1$. (This is obtained using the fact that the derived covering graph is the generalized Petersen graph.) Consider now the automorphism $\alpha=(134)(527)$ which must lift since an edge-transitive group of $\Gamma$ projects. Thus by [21, Theorem 4.2], the induced mapping $\bar{\alpha}$ must extend to an automorphism $x \mapsto \lambda x$ of $K$ (see the above discussion). As $\bar{\alpha}$ maps $\zeta\left(C_{j}\right)$ to $\zeta\left(C_{j}^{\alpha}\right)$, where $j \in\{1, \ldots, 5\}$, we get the following system of equations in $K$ :

$$
\lambda=a, \quad \lambda a=1, \quad \lambda a=a, \quad \lambda a=-4 a-1, \quad \lambda(a+1)=2 a .
$$

This gives $\lambda=a=1$ and $6=0$ in $K$, implying that $K$ is isomorphic to $\mathbb{Z}_{2}, \mathbb{Z}_{3}$, or $\mathbb{Z}_{6}$. If $K=\mathbb{Z}_{2}$ then $\Gamma \cong \operatorname{GP}(8,5) \cong \operatorname{GP}(8,3)$. If $K=\mathbb{Z}_{3}$ then $\Gamma \cong \operatorname{GP}(12,5)$. If $K=\mathbb{Z}_{6}$ then $\Gamma \cong \mathrm{GP}(24,5)$. 
Let $Y$ be the Petersen graph. Denote its vertices by $\{0,1,2,3,4,5,6,7,8,9\}$ in such a way that 012340 and 567895 are the outer and the inner cycles, respectively, and $05,16,27,38$, and 49 are the spokes. Choose a spanning tree consisting of edges $01,12,23,34,05,16,27,38$, and 49 . The base homology cycles are $C_{1}=401234$, $C_{2}=572105, C_{3}=683216, C_{4}=794327, C_{5}=8501238$, and $C_{6}=9612349$. Voltages on the spanning tree are 0 . Without loss of generality, we can assume that the voltages on the remaining arcs are $\zeta(4,0)=1$, and $\zeta(5,7)=\zeta(6,8)=\zeta(7,9)=a$, and $\zeta(8,5)=\zeta(9,6)=a+1$. (This is obtained using the fact that the derived covering graph is the generalized Petersen graph.) Consider now the automorphism $\alpha=$ (154)(289)(367) which must lift since an edge-transitive group of $\Gamma$ projects. Since the induced mapping $\bar{\alpha}$ must extend to an automorphism $x \mapsto \lambda x$ of $L$, and also $\bar{\alpha}$ maps $\zeta\left(C_{j}\right)$ to $\zeta\left(C_{j}^{\alpha}\right)$, where $j \in\{1, \ldots, 6\}$, we get the following system of equations in $L$ :

$$
\begin{aligned}
& \lambda=-2 a-1, \quad \lambda a=a, \quad \lambda a=5 a+2, \\
& \lambda a=a, \quad \lambda(a+1)=-3 a-1, \quad \lambda(a+1)=-3 a-1 .
\end{aligned}
$$

This gives $2=0, \lambda=-1$ and either $a=0$ or $a=1$ in $K$, implying that $K$ is isomorphic to $\mathbb{Z}_{2}$. If $a=0$ then $\Gamma \cong \mathrm{GP}(10,2)$. If $a=1$ then $\Gamma \cong \mathrm{GP}(10,7) \cong$ $\mathrm{GP}(10,3)$, completing the proof of Theorem 6.1 .

Acknowledgments István Kovács, Aleksander Malnič, Dragan Marušič, and Štefko Miklavič are supported in part by “Javna agencija za raziskovalno dejavnost Republike Slovenije,” Program No. P1-0285.

\section{References}

1. Cameron, P.J.: Problems from the fifteenth British combinatorial conference. Discrete Math. 167/168, 605-615 (1997)

2. Cameron, P.J.: Finite permutation groups and finite simple groups. Bull. Lond. Math. Soc. 13, 1-22 (1981)

3. Cameron, P.J., Giudici, M., Kantor, W.M., Jones, G.A., Klin, M.H., Marušič, D., Nowitz, L.A.: Transitive permutation groups without semiregular subgroups. J. Lond. Math. Soc. 6, 325-333 (2002)

4. Dobson, E.: Automorphism groups of metacirculant graphs of order a product of two distinct primes. Comb. Probab. Comput. 15, 105-130 (2006)

5. Dobson, E., Malnič, A., Marušič, D., Nowitz, L.A.: Minimal normal subgroups of transitive permutation groups of square-free degree. Discrete Math. 307, 373-385 (2007)

6. Dobson, E., Malnič, A., Marušič, D., Nowitz, L.A.: Semiregular automorphisms of vertex-transitive graphs of certain valencies. J. Comb. Theory B 97, 371-380 (2007)

7. Eves, H.: Elementary Matrix Theory. Allyn and Bacon Inc., Boston (1966)

8. Fang, X.G., Praeger, C.E.: Finite two-arc transitive graphs admitting a Suzuki simple group. Commun. Algebra 27, 3727-3754 (1999)

9. Feng, Y.Q., Kwak, J.H., Wang, K.S.: Classifying cubic symmetric graphs of order $8 p$ or $8 p^{2}$. Eur. J. Comb. 26, 1033-1052 (2005)

10. Frucht, R., Graver, J.E., Watkins, M.E.: The groups of the generalized Petersen graphs. Proc. Camb. Philos. Soc. 70, 211-218 (1971)

11. Giudici, M.: Quasiprimitive groups with no fixed point free elements of prime order. J. Lond. Math. Soc. 67, 73-84 (2003)

12. Giudici, M.: New constructions of groups without semiregular subgroups. Commun. Algebra 35, 2719-2730 (2007)

13. Giudici, M., Xu, J.: All vertex-transitive locally-quasiprimitive graphs have a semiregular automorphism. J. Algebraic Comb. 25, 217-232 (2007) 
14. Godsil, C.: Algebraic Combinatorics. Chapman \& Hall, New York (1993)

15. Gross, J.L., Tucker, T.W.: Generating all graph coverings by permutation voltage assignment. Discrete Math. 18, 273-283 (1977)

16. Gross, J.L., Tucker, T.W.: Topological Graph Theory. Wiley-Interscience, New York (1987)

17. Knapp, W.: On Burnside's method. J. Algebra 175, 644-660 (1995)

18. Kutnar, K., Šparl, P.: Distance-transitive graphs admit semiregular automorphisms. Eur. J. Comb. 31, 25-28 (2010)

19. Li, C.H.: The finite primitive permutation groups containing an abelian regular subgroup. Proc. Lond. Math. Soc. 87, 725-747 (2003)

20. Li, C.H., Lu, Z.P., Zhang, H.: Tetravalent edge-transitive Cayley graphs with odd number of vertices. J. Comb. Theory B 96, 164-181 (2006)

21. Malnič, A.: Group actions, coverings and lifts of automorphisms. Discrete Math. 182, 203-218 (1998)

22. Malnič, A., Nedela, R., Škoviera, M.: Lifting graph automorphisms by voltage assignments. Eur. J. Comb. 21, 927-947 (2000)

23. Marušič, D.: On vertex symmetric digraphs. Discrete Math. 36, 69-81 (1981)

24. Marušič, D.: Half-transitive group actions on finite graphs of valency 4. J. Comb. Theory B 73, 41-76 (1998)

25. Marušič, D., Scapellato, R.: Permutation groups, vertex-transitive digraphs and semiregular automorphisms. Eur. J. Comb. 19, 707-712 (1998)

26. Marušič, D.: On 2-arc-transitive Cayley graphs. J. Comb. Theory B 87, 162-196 (2003)

27. Müller, P. : Permutation groups with a cyclic two-orbits subgroup and monodromy groups of Siegel functions. arXiv:math/0110060v1[math.GR]

28. Oh, J.M., Wang, K.W.: Construction of one-regular graphs of valency 4 and 6. Discrete Math. 278, 195-207 (2004)

29. Praeger, C.E.: Finite normal edge-transitive Cayley graphs. Bull. Aust. Math. Soc. 6, 207-220 (1999)

30. Praeger, C.E.: Quasiprimitivity: structure and combinatorial applications. Discrete Math. 264, 211-224 (2003)

31. Schmidt, B.: Characters and Cyclotomic Fields in Finite Geometry. Lecture Notes in Mathematics, vol. 1797. Springer, Berlin (2002)

32. Wielandt, H.: Finite Permutation Groups. Academic Press, New York (1964) 